\title{
X-Ray Study on the Structure of the Poly(vinyl alcohol)-Iodine Complex
}

\author{
Yoon-So CHOI and Keizo MiYASAKA \\ Department of Organic and Polymeric Materials, Tokyo Institute of Technology, \\ Ookayama, Meguro-ku, Tokyo, Japan
}

(Received December 21, 1990)

\begin{abstract}
One-dimensional Fourier transform technique of $\mathrm{X}$-ray meridional intensity is applied to lightly iodinated samples in which the PVA-iodine complex is formed only in the amorphous phase. Thus calculated distribution function showed that a series of peaks with $3.1 \AA$ periodicity appears in much longer distance than expected from that due to $\mathrm{I}_{3}{ }^{-}$or $\mathrm{I}_{5}{ }^{-}$as suggested by Raman data. The values of the lattice size (Lc) and degree of disorder ( $\mathrm{g}$ ) were also estimated from Hosemann plots as $L c=31 \AA, g=0.052$. All these results from X-ray methods strongly suggest that linear polyiodine chains corresponding to $\mathrm{I}_{3}{ }^{-}$or $\mathrm{I}_{5}{ }^{-}$might be located so as to interfere with each other.
\end{abstract}

KEY WORDS Poly(vinyl alcohol)-Iodine Complex / Amorphous Phase /

Polyiodine / X-ray / Fourier Transform / Hosemann Plot /

We have so far studied the effects of PVA film structures, iodine concentration and temperature of soaking solutions on the formation and properties of PVA-iodine complex. $^{1-6}$ The effect of iodine concentration in the soaking solution on the complex formation is so strong that the complex formation should be studied separately at low and high iodine concentrations. At low concentrations the complex forms only in the amorphous phase, while at high concentrations the complex forms not only in the amorphous phase but also in the crystal phase. ${ }^{5,6}$ The width of X-ray meridional diffraction peak due to polyiodines which appears in the drawn PVA film soaked in the iodine solution was found much smaller than that expected from linear polyiodine corresponding to $\mathrm{I}_{5}{ }^{-}$or $\mathrm{I}_{3}{ }^{-}{ }^{7,8}$ These numbers of iodine atoms in a complex are those estimated from Raman and visible absorption spectra. ${ }^{4,9-13}$ This discrepancy implies that the number of iodine atoms forming a linear lattice is larger than 5 or 3 estimated from other techniques. One of the reasons for this discrepancy might be error in the X-ray analysis.

In the present paper, the coherent size of iodine lattice in lightly iodinated samples in which the complexes are formed only in the amorphous phase was studied in detail by one-dimensional Fourier transform of X-ray meridional intensity. This method is considered best to study this problem.

The effects of drawing and the degree of hydration of PVA films on the number of the polyiodine are also investigated using this method.

\section{EXPERIMENTAL}

PVA (Wako Pure Chem. Co., DP=2000) was used after saponification with sodium methoxide in methanol. The IR spectrum of the PVA showed no trace of acetate groups. Films were cast from a $7 \mathrm{wt} \%$ PVA aqueous solution on PMMA plates in air at $20^{\circ} \mathrm{C}$. The films with different degrees of orientation and hydration (volume fraction of water at the swelling equilibrium) were prepared by uniaxial drawing of the cast films at $120^{\circ} \mathrm{C}$ and 
subsequent annealing under fixed length at $150^{\circ} \mathrm{C}$ for $3 \mathrm{~min}$.

Iodine and potassium iodide (Wako Pure Chem. Co., GR grade) were used as received. An iodine-potassium iodide aqueous solution with a mol ratio of $1: 2$ and an iodine concentration of $1 \times 10^{-2} \mathrm{~mol} \mathrm{l}^{-1}$ was prepared for soaking PVA films. The soaking was carried out in solution at $20^{\circ} \mathrm{C}$ for $24 \mathrm{~h}$.

All X-ray measurements were carried out with $C u-K_{\alpha}$ rays. The diffraction intensity was measured in a transmission mode using a Rigaku SG-7 diffractometer equipped with a graphite-monochromator and a scintillation counter. Photographs were taken by Ni-filtered rays using a Rigaku flat Laue camera.

\section{RESULTS AND DISCUSSION}

In general, the $\mathrm{X}$-ray scattering amplitude $\psi\left(S_{1}, S_{2}, S_{3}\right)$ is given by the Fourier transform of the electron density distribution function $\rho\left(X_{1}, X_{2}, X_{3}\right)$ in a given substance:

$$
\begin{aligned}
\psi\left(S_{1},\right. & \left.S_{2}, S_{3}\right) \\
= & \iiint \rho\left(X_{1}, X_{2}, X_{3}\right) \\
& \times \exp \left\{2 \pi i\left(S_{1} X_{1}+S_{2} X_{2}+S_{3} X_{3}\right)\right\} \\
& \times \mathrm{d} X_{1} \mathrm{~d} X_{2} \mathrm{~d} X_{3}
\end{aligned}
$$

where $\left(X_{1}, X_{2}, X_{3}\right)$ and $\left(S_{1}, S_{2}, S_{3}\right)$ are orthogonal coordinates in real and reverse space, respectively, and direction 3 corresponds to the meridian parallel to the orientation axis of the specimen.

The meridional intensity $\mathrm{I}\left(00 S_{3}\right)$ is given by

$$
\begin{aligned}
\mathrm{I}\left(0,0, S_{3}\right)= & \left|\psi\left(00 S_{3}\right)\right|^{2} \\
= & \iint Q\left(X_{3}+r\right) Q\left(X_{3}\right) \mathrm{d} X_{3} \\
& \times \exp \left(2 \pi i S_{3} r\right) \mathrm{d} r
\end{aligned}
$$

with

$$
Q\left(X_{3}\right)=\iint \rho\left(X_{1}, X_{2}, X_{3}\right) \mathrm{d} X_{1} \mathrm{~d} X_{2}
$$

$r$ is the distance in the $X_{3}$ direction. Fourier transform of the $\mathrm{X}$-ray meridional intensity $\mathrm{I}\left(0,0, S_{3}\right)$ gives a radial distribution function $P(r)$ averaged on the $X_{1}-X_{2}$ plane in the real space.

$$
\begin{aligned}
P(r) & =\int Q\left(X_{3}+r\right) Q\left(X_{3}\right) \mathrm{d} X_{3} \\
& =\int \mathrm{I}\left(0,0, S_{3}\right) \exp \left(-2 \pi i S_{3} r\right) \mathrm{d} S_{3}
\end{aligned}
$$

The quantity $P(r)$ gives important information; (1) the periodicity in the $X_{3}$ direction, and (2) size of the periodic structure. In this case, we are interested in the latter.

Figure 1 shows X-ray diffraction photographs of drawn PVA and iodinated PVA films taken with the incident beam normal to the film plane. At this comparatively lower lodine concentration, the diffraction pattern of the PVA crystal remained unchanged, while a new spot appeared on the meridian. This new meridional spot comes from the PVA-iodine complex formed in the amorphous phase of PVA and has been attributed to polyiodine linear lattice of a $3.1 \AA$ periodicity in the complex. ${ }^{14}$

Figure 2 shows $\mathrm{X}$-ray meridional intensity curves of the original (a) and iodinated (b) PVA films. Curve (c) is the residue after subtraction of (a) from (b). Thus curve (c) is considered that from polyiodines. The peak at $2 \theta=29^{\circ}$ corresponds to $3.1 \AA$, and the peak at $2 \theta=61^{\circ}$, corresponding to $1.55 \mathrm{~A}^{\circ}$, is the second order reflection of the $29^{\circ}$ peak.

Figure 3 shows a Fourier transform of the intensity curve (c), giving a distribution function $P(r) . P(r)$ has a series of peaks of a $3.1 \AA$ periodicity as expected. If $N$ iodine atoms form a linear lattice of $3.1 \AA$ periodicity oriented along the fiber axis of PVA, and if no correlation takes palce among these iodine lattices, $P(r)$ should have $(N-1)$ peaks of $3.1 \AA$ periodicity and no more. However, in Figure 3 , peaks continue to appear even in the range of $r$ from $\mathrm{I}_{3}{ }^{-}$or $\mathrm{I}_{5}{ }^{-}$. 


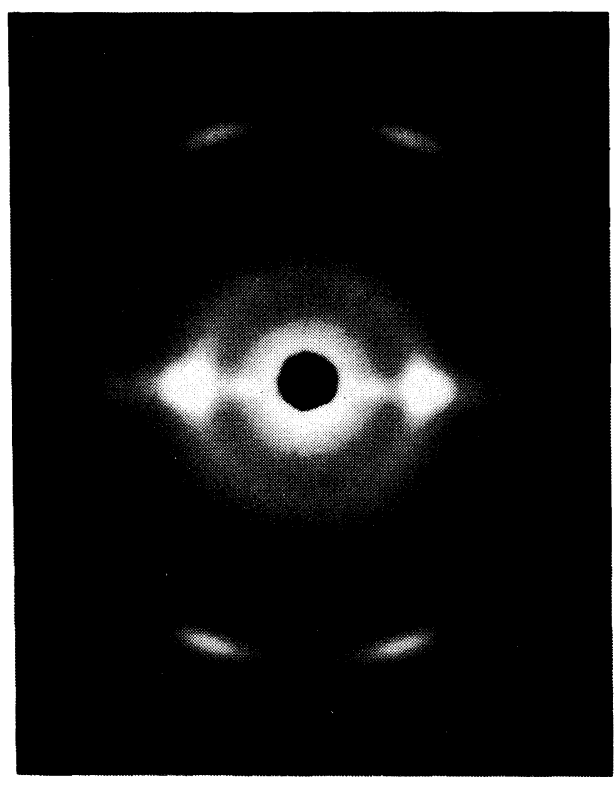

(a)

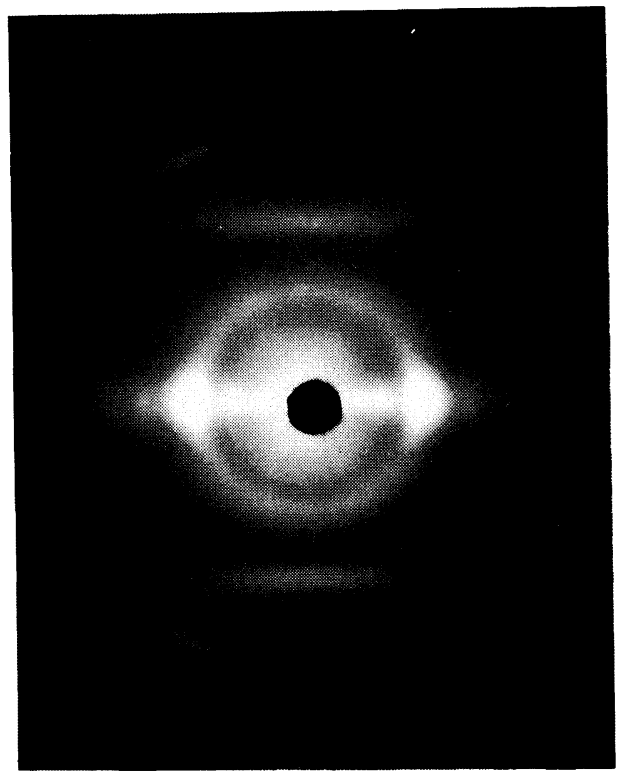

(b)

Figure 1. X-ray diffraction photographs of drawn PVA and iodinated PVA film taken with the incident beam normal to the film plane: (a) drawn PVA film $(\lambda=5)$ (b) iodinated PVA film soaked at $1 \times 10^{-2} \mathrm{~mol}^{-1}$

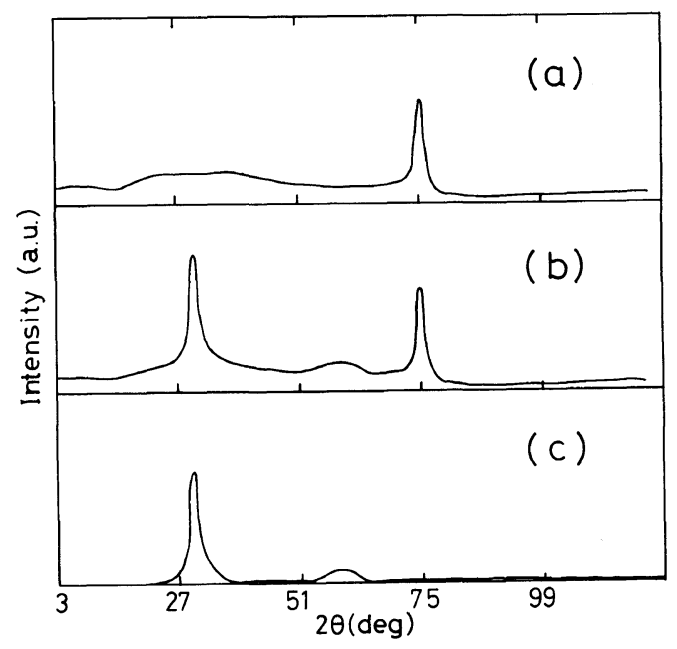

Figure 2. X-ray meridional intensity profiles: (a) drawn PVA film (b) iodinated PVA film (c) iodine ((b)-(a)).

In order to avoid the error due to separation of the intensity component of polyiodines (curve $\mathrm{c}$ in Figure 2), $P(r)$ was also estimated from the whole intensity (PVA + iodine) curve (curve $b$ in Figure 2). It should be noted that

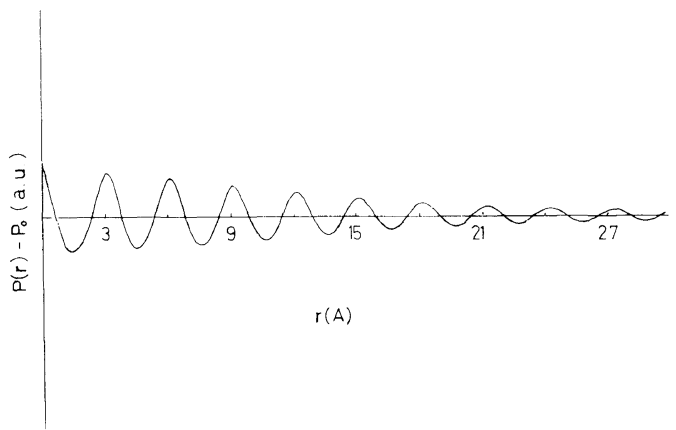

Figure 3. Distribution function $P(r)$ for polyiodines.

the periodicities of iodine and PVA (about $1.25 \AA$ ) are quite different, as shown in Figure 4. In this case also, there was no indication that a polyiodine is formed by 3 or 5 atoms.

Further, the size and degree of disorder of the lattice formed by polyiodine were evaluated using the Hosemann plot (eq 1) of the widths of the meridional diffractions due to polyiodine. 


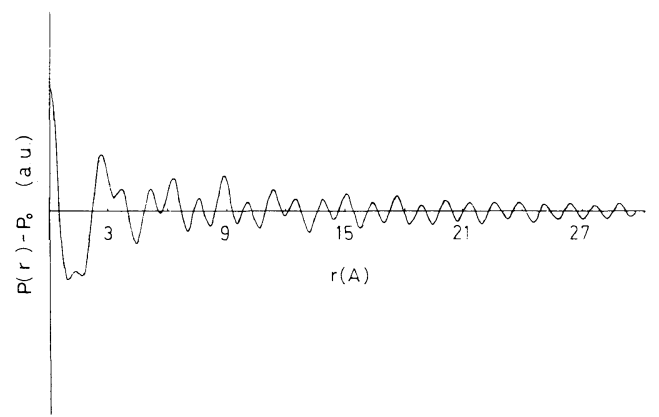

Figure 4. Distribution function $P(r)$ for iodinated PVA film.

$$
\left(\delta_{0}\right)^{2}=\frac{1}{L_{h k l^{2}}}+\frac{(\pi g)^{4} m^{4}}{d_{h k l^{2}}}
$$

where

$\delta_{0}$, width of the peak

$L_{h k l}$, mean dimension of the lattices

$d_{h k l}$, interplanar spacing

$g$, relative distance fluctuation

$m$, order of the reflection

We thus obtained $31 \AA$, corresponding to about 11 atoms, for the length of polyiodine, and 0.052 for the degree of disorder (nearly the same length was obtained by a simple application of Scherrer's equation to the first order meridional diffraction, because of the small disorder parameter).

These results strongly suggest that polyiodine chains consisting of 3 or 5 atoms exist not separately but coagulated to increase coherent length, resulting sharper diffraction than that expected from each chain.

Next, we investigated the effects of drawing and degree of hydration (D.H.) of PVA films on the number of iodine atoms in a polyiodine.

Figure 5 shows the peak intensity of $P(r)$ as a function of $r$ of iodinated PVA films with different draw ratio $\lambda$. Each peak intensity is standardized by that of the first peak. The peak intensity of $P(r)$ of two samples showed almost the same decay, suggesting that the structures of the polyiodine chain and complex are hardly

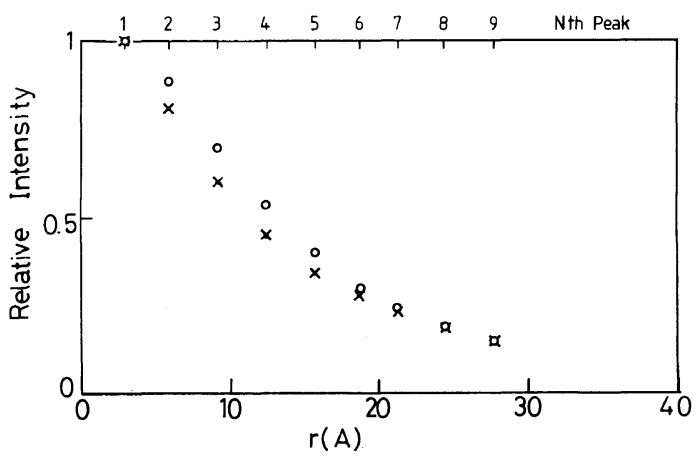

Figure 5. Effect of drawing of PVA films on $P(r)$. (O): $\lambda=3$. $(x): \lambda=5$.

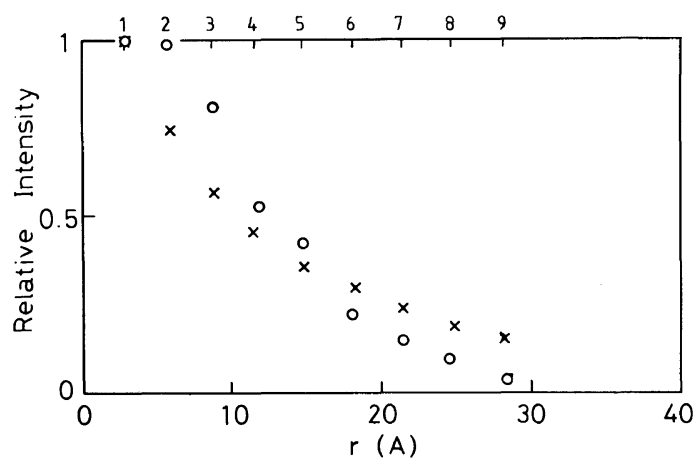

Figure 6. Effect of the degree of hydration of PVA films on $P(r)$. (O): D.H. $=0.58 .(\times)$ : D.H. $=0.82$.

affected by the drawing of PVA films.

Figure 6 shows the effect of D.H. of films on $P(r)$. The decay of $P(r)$ in the film with higher D.H. is slower than in the film with lower D.H.. This indicates that the number of iodine atoms in a polyiodine chain is larger in the higher D.H. film than in the lower D.H. one, or that the former polyiodines have better correlation with each other than the latter. It should be also noted that the second peak of $P(r)$ of the film with higher D.H. has the same degree of intensity as that of the first peak. This may be related to the difference in $\lambda_{\text {max }}$ in visible absorption. ${ }^{15}$ Kojima et al. ${ }^{1}$ reported that $\lambda_{\max }$ shifts continuously from 520 to $600 \mathrm{~nm}$ with increasing D.H., and concluded that the longer complex is formed in the film with higher D.H.. ${ }^{16-18}$ On the other hand, Oishi et al. ${ }^{4}$ concluded on the basis of resonance Raman 
spectra that only $\mathrm{I}_{5}{ }^{-}$is formed in iodinated swollen PVA films and that the shift of $\lambda_{\max }$ caused by increasing D.H. is due to structural change in the surroundings of the polyiodine chain. However, recent resonance Raman spectra ${ }^{13}$ using the $488.0 \mathrm{~nm}$ line of argon ion laser which is nearer $\lambda_{\max }$ of $\mathrm{I}_{3}{ }^{-}$than that of $\mathrm{I}_{5}{ }^{-}$showed that both $\mathrm{I}_{3}{ }^{-}$and $\mathrm{I}_{5}{ }^{-}$complexes are formed in the amorphous phase, supporting Kojima's conclusion. The effect of D.H. on the decay of $P(r)$ peak in Figure 6 may be related to increase in the fraction of the $\mathrm{I}_{5}{ }^{-}$complex.

\section{REFERENCES}

1. Y. Kojima, K. Furuhata, and K. Miyasaka, J. Appl. Polym. Sci., 30, 1617 (1985).

2. Y. Oishi and K. Miyasaka, Polym. J., 18, 307 (1986).

3. Y. Oishi and K. Miyasaka, Polym. J., 19, 331 (1987).

4. Y. Oishi, H. Yamamoto, and K. Miyasaka, Polym.
J., 19, 1261 (1987).

5. Y. S. Choi, Y. Oishi, and K. Miyasaka, Polym. J., 22, 601 (1990).

6. H. Sakuramachi, Y. S. Choi, and K. Miyasaka, Polym. J., 22, 638 (1990).

7. M. Haisa and H. Itami, J. Phys. Chem., 61, 817 (1957).

8. Y. S. Choi, Y. Oishi, and K. Miyasaka, Polym. Prep. Jpn., 37, 2615 (1988).

9. T. Yokota and Y. Kimura, Makromol. Chem., 185, 749 (1984).

10. T. Yokota and Y. Kimura, Makromol. Chem., 186, 549 (1985)

11. M. E. Heyde, L. Rimai, R. G. Kilponen, and D. Gill, J. Am. Chem. Soc., 94, 5222 (1972).

12. F. Inagaki, I. Harada, T. Shimanouchi, and M. Tasumi, Bull. Chem. Soc. Jpn., 45, 3384 (1972).

13. Y. S. Choi and K. Miyasaka, Polym. Prep. Jpn., 39, 2439 (1990)

14. C. D. West, J. Chem. Phys., 15, 689 (1947).

15. M. M. Zwick, J. Appl. Polym. Sci., 9, 2393 (1965).

16. F. Cramer and W. Herbst, Naturwissenschaften, 39, 259 (1952).

17. H. Murakami, J. Chem. Phys., 22, 367 (1954).

18. H. Pjojosubroto and Y. Tanizaki, Bull. Chem. Soc. Jpn., 43, 3025 (1970). 